\title{
Experimental Verification of Acoustic Saturation
}

\author{
J. M. Sempsrott and W. D. O'Brien, Jr. \\ Bioacoustics Research Laboratory, Department of Electrical and Computer Engineering, \\ University of Illinois, 405 N. Mathews, Urbana, IL 61801
}

Abstract -- The maximum value of acoustic pressure within an ultrasonic beam is limited by acoustic saturation. The theoretical basis for acoustic saturation has been analyzed previously. In this work, a high-power pulse source is used to drive acoustic pressure levels from an ultrasonic beam to saturation. An automatic procedure determines the field's beam axis using a calibrated Marconi PVDF hydrophone. The hydrophone measured RF waveforms are recorded along the beam axis and analyzed off-line. The peak compressional pressure is evaluated as a function of distance along the beam axis. The overall maximum pressure is compared to theoretical predictions. Three-, six-, and nine-MHz center frequency, 19-mm-diameter transducers were analyzed. The longer focal length transducer saturation level was comparable to a theoretical prediction. However, the theoretical saturation level for the shorter focal length transducers underestimated experimental results.

\section{INTRODUCTION}

The theory of acoustic saturation for a converging wave was developed in 1959 by Naugol'nykh and Romanenko [1]. The concept of acoustic saturation states that as the transducer's drive voltage increases there exists a finite limit of the acoustic pressure at the focus. This limitation occurs in pulsed diagnostic beams in the form of acoustic non-linearity, or acoustic shock. The theoretical pressure saturation equation, developed from [1], is [2]:

$$
P_{\text {sat }}=\frac{\rho_{o} c^{3}}{2(\beta) f F} \frac{G}{\ln (G)}
$$

where $\rho_{o}=$ equilibrium density, $c=$ speed of sound in the medium, $\beta=$ nonlinear propagation constant, $f=$ center frequency for the transducer, $F=$ focal length, and $G=$ focusing gain for the converging beam. An approximation for $G$ is given [2] as a relation between the transducer's Rayleigh distance, $z_{r}$, and the focal length $F$, as shown in equation (2). The Rayleigh distance is further described with a relation between the radius of the transducer, $a$, and the wavelength of the source pulse, $\lambda$ :

$$
G=\frac{z_{r}}{F}=\frac{\pi\left(a^{2}\right)}{\lambda F}
$$

The approximation for $G$ applies for small aperture angles[2], $\phi$ in Fig. 1, or long focal lengths compared to source diameters.

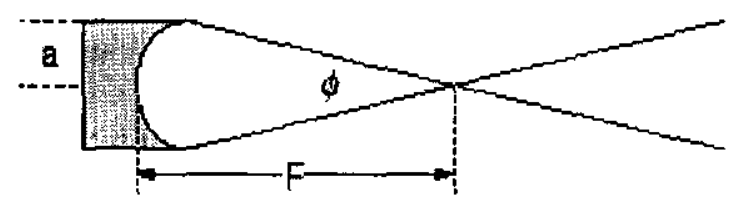

Fig. 1: Geometry for spherically focused transducer.

There are two main objectives to the work: 1) to develop experimental data that shows the presence of acoustic saturation and 2) to evaluate those results against values predicted from theory.

\section{PRocedure}

The AIUM Measurement and Labeling Standard [3] was the basis for finding the acoustic pressure values. The beam axis is a straight line from the transducer that connects points of measured maximum pulse intensity integrals $(P I I)$ at various positions from the transducer. $P I I$ can be described accordingly:

$$
P I I=\frac{1}{\rho c} \int P^{2}(t) d t
$$

where $P(t)$ is the RF pressure waveform and $\rho c$ is the characteristic acoustic impedance. To determine the beam axis, an automated procedure is used that employs a micropositioning system, an oscilloscope (LeCroy Model 9354TM), a high-powered, pulsed source (RAM5000, Ritec, Inc.), a PVDF hydrophone (Marconi, Ltd), transducers (Matec Instruments, Inc. and Panametrics, Inc.), a degassed water tank, and a controlling computer as seen in Fig. 2. 


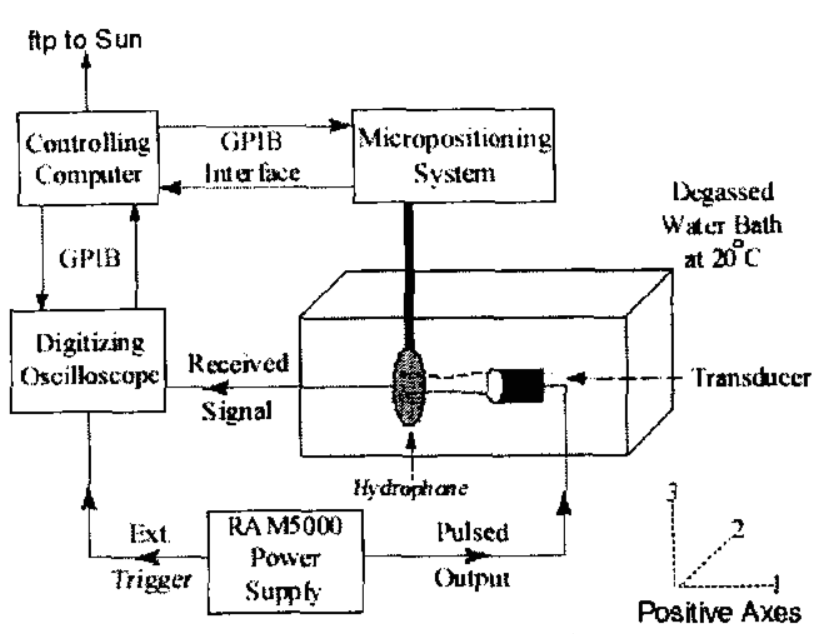

Fig. 2: Block diagram of system with positive axes convention indicated.

The entire procedure contains four steps: 1) manual setup, 2) automated determination of the maximum $P I I$ as a function of distance from the source for calculation of the beam axis, 3) collection of RF waveforms along the beam axis, and 4) calculation of the appropriate pressure values.

\section{Manual Setup}

A one-cycle electrical pulse is applied at the transducer's center frequency. The technique for determining the center frequency is described in [4]. Determination of the beam axis begins by manually finding the transducer's maximum signal as seen on the oscilloscope. The hydrophone is then moved 500 $\mu \mathrm{m}$ in both the negative axis- 2 and axis- 3 directions and moved $2 \mathrm{~mm}$ in the positive axis-1 direction. Offsetting the hydrophone along axes- 2 and -3 allows the automated procedure to scan those axes to where $P I I$ is maximized within that axis 2-3 plane. Moving the hydrophone in the positive axis-1 direction allows a max $P I I$ position to be found in the axis 2-3 plane between the transducer and the geometric focus. This will be the origin of the scan, or the $(0,0,0)$ point, where the coordinate designation is (axis 1 , axis 2 , axis 3 ).

\section{Determination of Beam Axis}

A total of 10 positions of maximum $P I I$ are determined during the scan process, five each for axes- 2 and -3 . Positions are collected every $2 \mathrm{~mm}$ along axis- $1,8 \mathrm{~mm}$ total in the axial direction. All coordinates are given in $\mathrm{mm}$ unless specified otherwise.
Starting from $(0,0,0)$, the hydrophone moves at 50 micron increments a total of $1 \mathrm{~mm}$ in the axis-2 direction in search of the signal with the maximum $P I I$, and then returns to $(0,0,0)$. The position where the $P I I$ was maximized is stored for later use. The same procedure is done for the axis-3 direction. Figure 3 shows hydrophone movements for axes- 2 and -3 .

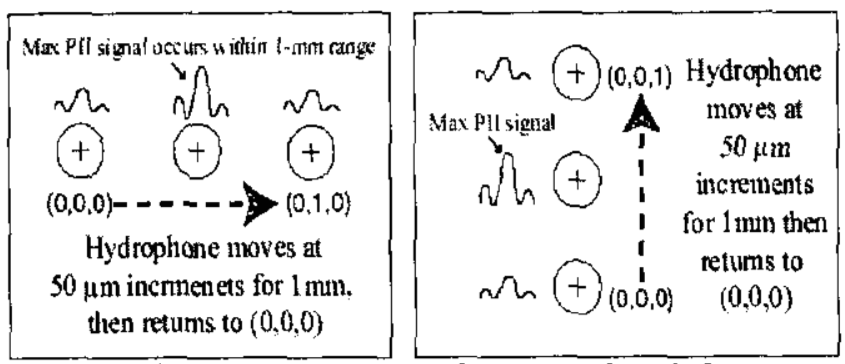

Fig. 3: Hydrophone movement along axes-2 and -3 .

Two positions exist where the $P I I$ is a maximum, one each for axis- 2 and -3 . The procedure in Fig. 4 is repeated at $(2,0,0),(4,0,0),(6,0,0)$, and $(8,0,0)$. The two positions found in each axis- 1 plane correspond to the axis- 2 and axis- 3 coordinates of the maximum $P I I$, as shown in Fig. 4.

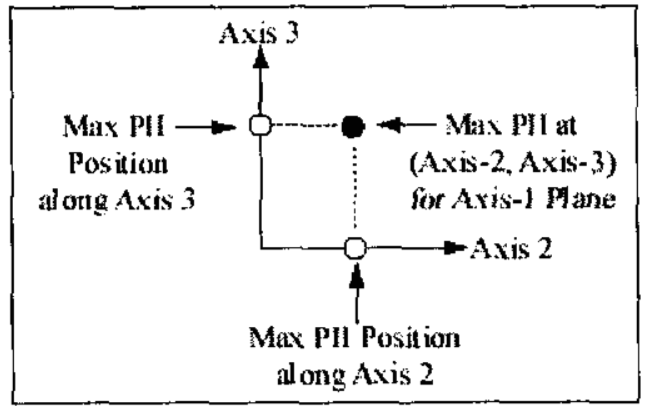

Fig. 4: Position of maximum PII along axis-1.

By definition of the beam axis [3], those 5 coordinates of maximum $P I I$ for each position along axis-1 can be used to create a best fit line that corresponds to the transducer's beam axis.

\section{Waveform Collection}

After the beam axis is calculated, the hydrophone moves back to $(0, x, y)$, where $x$ and $y$ are the beam axis positions in axis- 2 and -3 when axis- 1 is at zero. The hydrophone moves in $50-\mu \mathrm{m}$ increments along the beam axis, and collects RF waveforms at 500 $\mathrm{Ms} / \mathrm{s}$. The waveforms are transferred to a Sun UltraSparc workstation where they are analyzed using Matlab $^{\circledR}$. 


\section{Analysis of Collected Waveforms}

In Matlab ${ }^{\oplus}$, a program calculates pressure values using voltage-to-pressure conversions provided with the calibrated hydrophone. The maximum positive pressure, or compressional pressure $\left(p_{c}\right)$, is acquired from the waveforms, and plotted vs. distance from the transducer (Fig. 5).

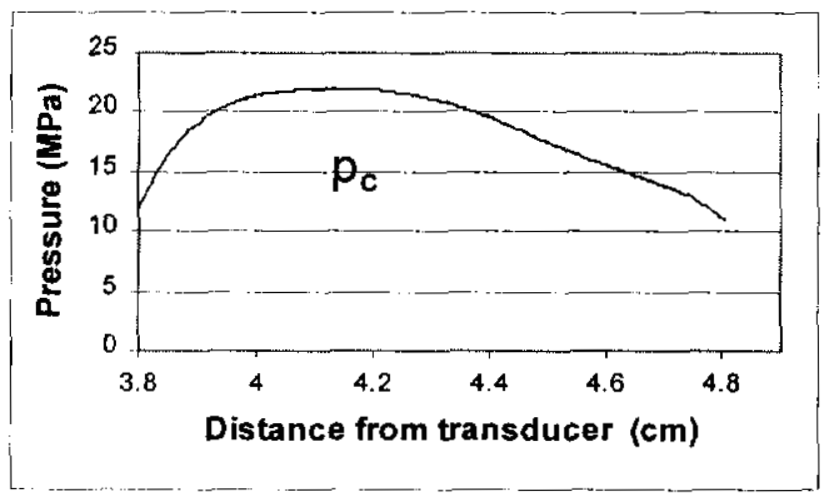

Fig. 5: Plot of $p_{c}$ vs. distance from transducer.

\section{II. RESULTS}

The transducers analyzed in this experiment were $19-$ mm-diameter, $9-\mathrm{MHz}, \quad 6-\mathrm{MHz}$, and $3-\mathrm{MHz}$ transducers. The two $9-\mathrm{MHz}$ transducers included focal lengths of $f / 3$ and $f / 2$, the two $6-\mathrm{MHz}$ transducers included an $f / 2$ and $f / 1$, and the $3-\mathrm{MHz}$ transducer was an $\mathrm{f} / 1$.

\section{Theoretical Values}

The theoretical saturation pressure values can be calculated directly using equations (1) and (2). The constant values used for equation (1) are $\rho_{o}=998$ $\mathrm{kg} \cdot \mathrm{m}^{-3}, c=1500 \mathrm{~m} / \mathrm{s}$, and $\beta=3.5$. The value for $\beta$ has been determined previously[5]. The center frequency, $f$, and focal length, $F$, depend on the transducer, and those values are found according to the wire-technique[4]. Those results are given in Table 1.

The value for $G$ is calculated using equation (2), where the radius, $a$, is a constant $9.5 \mathrm{~mm}$ for each transducer, and the wavelength, $\lambda$, is calculated from the measured frequency and the known speed of sound, $c$. The theoretical values for $G$ and $P_{\text {sat }}$ are also given in Table 1.
Table 1: Measured and calculated values for 5 transducers used.

\begin{tabular}{cccccc}
\hline $\begin{array}{c}\text { Nominal } \\
\mathbf{f}\end{array}$ & $\mathbf{f} / \#$ & $\begin{array}{c}\text { Measured } \\
\mathbf{f}\end{array}$ & $\begin{array}{c}\mathbf{F} \\
(\mathrm{MHz})\end{array}$ & & \multicolumn{2}{c}{ Calculated } \\
$(\mathrm{MHz})$ & $(\mathrm{mm})$ & $\mathrm{G}$ & $\begin{array}{c}\boldsymbol{P}_{\text {sat }} \\
(\mathrm{MPa})\end{array}$ \\
\hline 9 & 3 & $\mathbf{8 . 3 7}$ & 51.54 & 30.7 & 10.0 \\
9 & 2 & 8.23 & 39.35 & 39.5 & 16.0 \\
6 & 2 & 5.61 & 40.24 & 26.4 & 17.2 \\
6 & 1 & 5.58 & 20.88 & 50.5 & 53.2 \\
3 & 1 & 2.82 & 21.14 & 25.2 & 63.0 \\
\hline
\end{tabular}

Experimental Results

One of the main objectives of this work is to provide experimental data that indicates the effects of acoustic saturation. Five trials were performed on each transducer. The input voltage was varied from approximately $0.1 \mathrm{kV}$ up to $2 \mathrm{kV}$. At each increment, the procedure for finding the beam axis was done and the corresponding data was analyzed for maximum $p_{c}$ values. Those maximum $p_{c}$ values were then plotted vs. the input voltage to the transducer, as shown in Figs. 6(a)-(c). Also indicated on the plots is the theoretical saturation level for each transducer, as calculated from equation (1).

From Fig. 6(a) the effects of acoustic saturation can clearly be seen. The experimental results for the 9 $\mathrm{Hz} \mathrm{f} / 3$ appear to be approaching the predicted saturation level of $10 \mathrm{MPa}$. The results for the $9-\mathrm{Hz}$ $f / 2$ show that the predicted saturation level underestimates the experimental results by more than $20 \%$.

The remaining results do not show signs of saturation. Fig.6(b) shows that when the $6-\mathrm{MHz} \mathrm{f} / 2$ transducer does reach its saturation limit it will be above the predicted value for saturation. Results obtained for the $f / 1$ transducers have not reached the saturation level.

The results from the $9-\mathrm{MHz}$ transducers do agree with equation (1) in that the longer focal length transducer saturated at a lower pressure level. In the case of the $6-\mathrm{MHz}$ transducer, the $\mathrm{f} / 1$ transducer was reaching much higher pressure levels than the $f / 2$, which would indicate that the saturation for the 6$\mathrm{MHz} \mathrm{f} / 1$ will also be higher than the $\mathrm{f} / 2$ transducer.

In looking at both $\mathrm{f} / 1$ transducers, the $3-\mathrm{MHz}$ reached a higher pressure level than the 6-MHz. This would indicate that the saturation levels would follow the same trend, which also agrees with equation (1). 


\section{CONCLUSIONS}

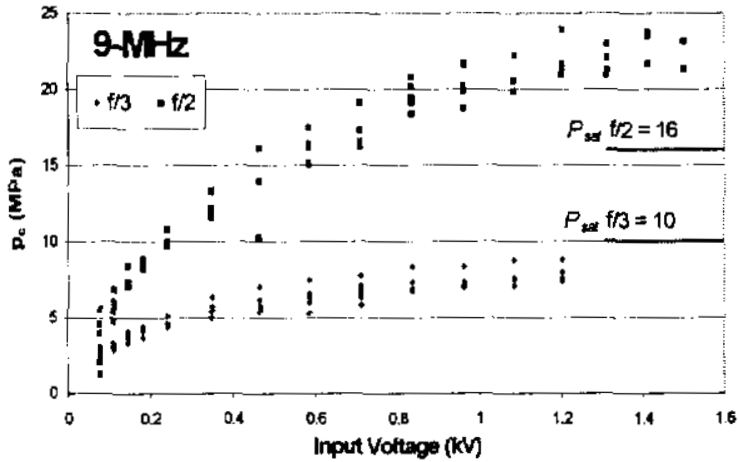

Fig. 6(a): $p_{c}$ vs. Input Voltage for the 9-MHz transducers. $P_{\text {sat }}$ levels for each are also indicated.

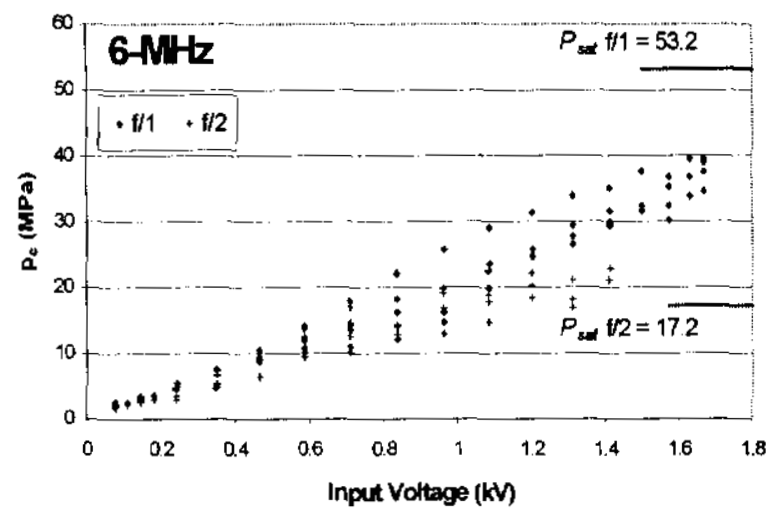

Fig. 6(b): $\mathrm{p}_{\mathrm{c}}$ vs. Input Voltage for the 6-MHz transducers. $P_{\text {sat }}$ levels for each are also indicated.

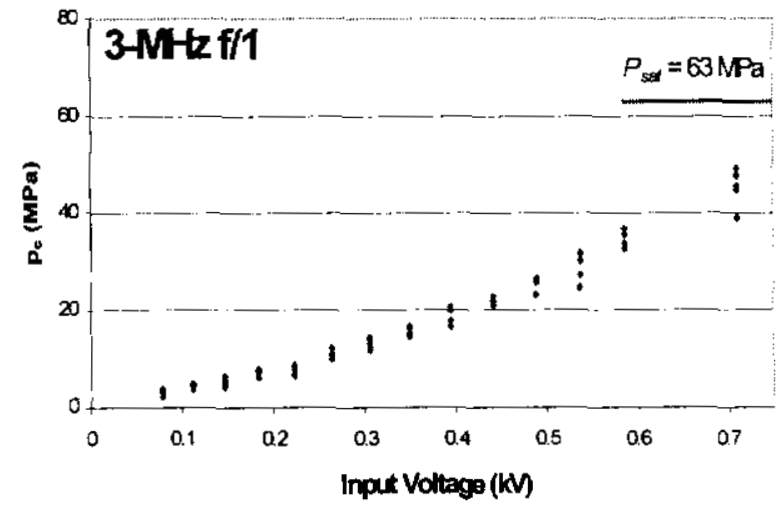

Fig. 6(c): $\mathrm{p}_{\mathrm{c}}$ vs. Input Voltage for the 3-MHz transducer. The $P_{\text {sat }}$ level is indicated.
The results from the $9-\mathrm{MHz} \mathrm{f} / 3$ and $\mathrm{f} / 2$ transducers verify acoustic saturation. The remaining transducers did not appear to saturate. The results agree with the theory of equation (1) in that transducers of the same frequency and longer focal length reach saturation at lower pressures. Also, for transducers of the same focal length, the lower frequency transducers will saturate at higher pressure levels.

The underestimation of the actual saturation levels may be attributed to the estimation in $G$, the focusing gain from equation (2). The results show that for long focal lengths $(9-\mathrm{MHz} / / 3)$, the estimation for $\mathrm{G}$ is adequate, and theoretical calculations are comparable to experimental results. However, decreasing the focal length, which increases the value for $G$, causes the theory to underestimate experimental results.

K. A. Naugol'nykh and E. V. Romanenko, "Amplification factor of a focusing system as a function of sound intensity," Soviet Physics-Acoustics, vol. 5, pp. 191-195, 1959.

F. A. Duck, "Acoustic saturation and output regulation," Ultrasound in Medicine and Biology, vol. 25, No. 6, pp. 1009-1018, 1999.

"Acoustic Output Measurement and Labeling Standard for Diagnostic Ultrasound Equipment," AIUM Publications, American Institute of Ultrasound in Medicine, 11200 Rockville Pike, Suite 205, Rockville, MD 20852, 1992.

[4] K. Raum and W. D. O'Brien, Jr., "Pulse-echo field distribution measurement technique for high-frequency ultrasound sources," IEEE Trans. Ultrason., Ferroelect., Freq. Contr., vol. 44, pp. 810-815, July 1997.

[5] M. F. Hamilton and D. T. Blackstock (eds.), "Nonlinear Acoustics," San Diego: Academic Press, 1998, pp. 25-39. 